Frequency of Bone Fractures among Hospitalized Patients Injured by the Bam Earthquake

A. Hosseinnezhad; A. Bidari; $M$. Zare; A. Nejati;

M. Tahani; S. Abbasi; M. Jallili; M. Ahangar

Iran University Of Medical Sciences, Iran

Introduction: On 26 December 2003, a devastating earthquake destroyed Bam, a city in southeastern Iran. After this event, a database was provided by a large prospective multicenter, cross-sectional study, which recruited all injured patients who remained hospitalized in five different provinces of Iran one week after the earthquake.

Objective: To determine the frequency of different types of bone fractures in hospitalized earthquake victims.

Method: Pertinent data including gender, age, and specific types of radiologically proven bone fractures were extracted from the original database.

Results: Of a total of 1,052 hospitalized earthquake victims, $484(46.01 \%)$ were female and 568 (53.99\%) were male. Collectively, $692(65.8 \%)$ patients had radiologically proven fractures. The fractures were categorized into three groups. Those fractures affecting the long bones of upper and lower extremities were diagnosed in 395 cases $(37.5 \%$ of the total cases). Pelvic fractures were detected in 264 cases (25.1\% of the total cases), and vertebral fractures were found in 124 cases (11.8\% of the total cases). A total of 91 patients were grouped in at least two of the three categories. Respectively, $348(71.9 \%)$ and $344(60.5 \%)$ of female and male patients had proven fractures, with mean ages of 28.9 and 29.9 years.

Conclusion: Bone fractures accounted for one of the most common injuries in earthquake victims and were more prevalent in the long bones of extremities compared to the pelvis and spine. Females noticeably were more prone to sustain bone fractures than men, probably due to their lower bone density.

Keywords: Bam; bone fractures; earthquakes; gender; injuries; Iran; patients

Prebosp Disast Med 2005;20(3):s145

\section{Prevalence of Acute Renal Failure in Hospital Patients Injured in the Bam Earthquake}

A. Bidari; A. Hosseinnezhad; M. Zare; H. Hatamabadi; $S$. Farabmand; A. Arbami; M. Talebian; F. Bozorgi

Iran University Of Medical Sciences, Iran

Introduction: Acute renal failure (ARF), mainly as a consequence of rhabdomyolysis, has been known to be a major medical complication in earthquake victims.

Objective: To evaluate the incidence of ARF and its causal factors in hospitalized victims of the Bam earthquake on 26 December 2003 in southeastern Iran.

Methods: In a prospective, multi-center, cross-sectional study, data were collected from 1,052 patients injured in the Bam earthquake, who were still hospitalized one week after the devastating earthquake occurred. Acute renal failure was defined as a serum creatinine level of $\geq 3 \mathrm{mg} / \mathrm{dL}$ detected within the first week after the earthquake.

Results: A total of $10.6 \%$ of the hospitalized patients, with a mean age of 28.4 years, fulfilled the study criteria for
ARF. Among those affected by ARF, $34.5 \%$ were female and $65.5 \%$ were male. A total of $71.2 \%$ of the ARF cases underwent hemodialysis. The median creatine phosphokinase (CPK) levels obtained for the ARF group after admission was $11,300 \mathrm{u} / \mathrm{L}$ (range: $132-500,000 \mathrm{u} / \mathrm{L}$ ) and the median peak CPK documented during the first week after injury was $19,930 \mathrm{u} / \mathrm{L}$ (range: $598-500,000 \mathrm{u} / \mathrm{L}$ ).

The mean admission of Blood Urea Nitrogen (BUN) was $125 \pm 14.2 \mathrm{mg} / \mathrm{dL}$, and the peak level of BUN in ARF cases was $139 \pm 13.5 \mathrm{mg} / \mathrm{dL}$. The mean creatinine on admission was $6.04 \pm 0.58 \mathrm{mg} / \mathrm{dL}$ and the mean maximum creatinine in ARF cases was $6.86 \pm 0.52 \mathrm{mg} / \mathrm{dL}$. The mean first CPK in cases requiring hemodialysis was 51,184 $\pm 26,430 \mathrm{u} / \mathrm{L}$, while in non-hemodialysis cases it was $28,736 \pm 15,500 \mathrm{u} / \mathrm{L}$. Patients who underwent hemodialysis were trapped in the rubble a mean of $5.7 \pm 0.99$ hours in comparison with $4.75 \pm 4.6$ hours in patients not requiring hemodialysis.

Conclusion: A considerable number of earthquake victims developed ARF, mainly due to rhabdomyolysis, in the first week following the event. Most of them underwent hemodialysis as a life-saving modality. Optimal hydration should be considered as a major prophylactic measure to reduce the likelihood of developing ARF in earthquakeinjured patients.

Keywords: acute renal failure (ARF); earthquake; hemodialysis Prebosp Disasi Med 2005;20(3):s145

\section{Patients with Multiple Traumatic Injuries with Hemorrhagic Shock Treated with IVR Y. Minagawa \\ Kuji Hospital, Japan}

Introduction: The characteristics and treatment for victims with multiple traumatic injuries are changing, and more exact medical treatments are being required.

Methods: The study was based on 20 cases, involving treatment with IVR from 1998 to 2004. For each case, blood pressure, the presence of shock, period of hospitalization, the use of arterial embolization, and survival were examined. Additionally, age, gender, alterations in consciousness, the time from injury to IVR, and the length of the hospital stay were abstracted.

Results: The averages of the ages of the patients was 51 years, and 15 men and four women were included in the study. The injured studied were results of eight traffic crashes, two industrial accidents, eight falls from height, and one other case. The average of the transport times was $60 \mathrm{~min}$ utes and of the times to IVR ranged from 46 minutes to 48 hours. Blood pressure ranged from $80-160 \mathrm{mmHg}$, with an average of $114 \mathrm{mmHg}$. Six cases presented with shock. The length of stay ranged from 0-87 days, with an average of 23 days. Arterial embolization procedures included four to the liver, two to renal arteries, four to the iliac arteries, and one to the inferior diaphragmatic arteries. Four cases died due to hemorrhagic shock.

Conclusion: In the case of patients with multiple traumatic injuries, IVR was considered to be useful as a quick and exact diagnostic medical treatment. 
Keywords: arterial; diagnosis; embolization; IVR; shock; trauma; treatment

Prehosp Disast Med 2005;20(3):s145-s146

Comparison of Abdominal X-ray and CT Scan in 56 Opium and Heroin Body Packers in Mashhad, Iran M. Balali-Mood; G. Zare

Mashhad University of Medical Sciences, Iran

Introduction: Opium and heroin body packing is a social and health problem in Mashhad since the city is in the vicinity of the Afghanistan border.

Objective: To study health aspects of the body packers admitted in Imam Reza Toxicology Ward between 2001 and 2004 and to compare abdominal X-ray (AXR) and computed tomography (CT) scan for the diagnosis of the patients.

Methods: All body packers referred to the Toxicology Ward of Imam Reza Hospital in Mashhad, Iran between 2001 and 2003 were studied. They were hospitalized and underwent close observation for any sign of intoxication. An AXR and a urine morphine test were performed for all patients. An abdominal CT scan also was carried out for 41 of these patients. Naloxane and supportive care were applied to those with clinical manifestations of intoxication. AXR and CT scan results were classified into three groups (highly suggestive, suggestive, and false positive) and compared, using the chi-square test.

Results: A total of 56 body packers (54 males and two females) aged $32.1 \pm 11.3$ years were studied. A mean of $44.4 \pm 35.1$ opium and $52.0 \pm 20.0$ heroin packets weighing 8-15 grams were retrieved from 46 and 10 patients, respectively. Most of the patients (43\%) were uneducated, while $32 \%, 23 \%$, and $2 \%$ of them had primary, secondary, and university education, respectively. The majority of the patients $(71 \%)$ were married, but only $25 \%$ of them were employed. The highest frequency of admissions was in autumn (18 patients) followed by winter (17 patients) and summer ( 15 patients). Twenty-six patients were unconscious at the time of admission and six of these were admitted to the intensive care unit (ICU). All unconscious patients responded well to Naloxane. The mean period of hospitalization was $4.7 \pm 2.7$ days for opium and $4.1 \pm 0.6$ days for heroin packers. The urine morphine test was positive in $82 \%$ of the patients. Nine patients underwent surgical operation and death occurred in only three opium packers. As shown in the table, AXR and CT scan results were significantly different $\left(\chi^{2}=46.1\right.$, diff $\left.=2, p<0.001\right)$.

Considering defecation of the packets as the gold standard diagnostic method, sensitivity of AXR and CT scan in diagnosis of body packing was determined as $92 \%$ and $100 \%$, respectively.

Conclusions: Although AXR is a simple and more available technique for the diagnosis of opium and heroin body packing, CT scan was shown to be a more significant sensitive diagnostic tool and should be employed as the confirmatory method.

\begin{tabular}{|l|c|c|}
\hline \multicolumn{1}{|c|}{ Results } & \multicolumn{2}{c|}{ Diagnostic Methods } \\
\hline & Abdominal X-ray & Abdominal CT Scan \\
\hline Highly Suggestive & 16 & 40 \\
\hline Suggestive & 36 & 1 \\
\hline False Negative & 4 & 0 \\
\hline Total & $\mathbf{5 6}$ & $\mathbf{4 1}$ \\
\hline
\end{tabular}

Table 1-Comparison of abdominal X-ray and CT scan results in diagnosis of body packing $(\mathrm{CT}=$ computed tomography)

Keywords: body packing; CT scan; diagnosis; heroin; opium; patients; $x$-ray

Prebosp Disast Med 2005;20(3):s146

\section{Use of Hypnosis in General Emergency Service}

F. van Trimpont

$\mathrm{CH}$ Hornu-Frameries, Belgium

Introduction: Hypnosis can provide an interessant support to analgesia and relaxation of patient in a general emergency service. Indications for and feasibility of using hypnosis in such circumstances are reported.

Methods: Two indications for using hypnosis were selected. First, it can provide help to manage analgesia. Hypnosis generally can be practiced with children before realization of other techniques (IV line, local or regional anesthesia). Children are more relaxed after hypnosis and therefore respond better to medical examinations and techniques. This indication also is useful for anxious adults as a secondary technique to complement analgesia. A second indication of using hypnosis is relaxation for patients who need non-invasive, respiratory techniques. These patients generally have problems breathing into such apparatuses and an easy technique, like hypnosis, which doesn't modify respiratory rates, helps patients accept non-invasive, respiratory techniques and avoid intubation.

Conclusion: The use of hypnosis in an emergency service can help patients accept some techniques used in emergency situations and also can allow patients to be more relaxed and have a better experience with analgesia, especially for children.

Keywords: analgesia; children; emergency services; hypnosis; indications; relaxation

Prehosp Disast Med 2005;20(3):s146

\section{New Criteria for Deciding Damage Control Surgery for Severe Abdominal Trauma}

K. Mashiko; Y. Sakamoto; H. Matsumoto; K. Takubiro; Y. Hara; R. Morita; K. Takei; Y. Ueno; K. Abe; Y. Tomita

Chiba-Hokusoh Hospital, Japan

Purpose: As a standard, factors that determine the need for damage control surgery (DCS) include the deadly triad of acidosis, hypothermia, and coagulopath. However confirmation of the coagulopathy requires time and it might not be possible to utilize the triad in the emergency room (ER) setting. Therefore, the simple and practical criteria for the DCS (hypotension, hypothermia, and acidosis) in victims of severe abdominal trauma were evaluated. 